\title{
ON THE TAUTOLOGICAL RING OF A JACOBIAN MODULO RATIONAL EQUIVALENCE
}

\author{
BAOHUA FU AND FABIEN HERBAUT
}

\begin{abstract}
We consider the Chow ring with rational coefficients of the Jacobian of a curve. Assume $D$ is a divisor in a base point free $g_{d}^{r}$ of the curve such that the canonical divisor $K$ is a multiple of the divisor $D$. We find relations between tautological cycles. We give applications for curves having a degree $d$ covering of $\mathbb{P}^{1}$ whose ramification points are all of order $d$, and then for hyperelliptic curves.
\end{abstract}

\section{INTRODUCTION}

1.1. For an abelian variety $X$ of dimension $g$, we denote by $C H(X)=$ $\oplus_{p=0}^{g} C H^{p}(X)$ the Chow ring of $X$ with rational coefficients. Let $k: X \rightarrow X$ be the morphism $x \mapsto k x$ for any $k \in \mathbb{Z}$. It turns out that all the pull-backs $k^{*}$ and push-forwards $k_{*}$ on the level of Chow rings can be diagonalized simultaneously, which gives the following decomposition ([B2]):

$$
C H^{p}(X)=\bigoplus_{i=p-g}^{p} C H_{(i)}^{p}(X),
$$

where $C H_{(i)}^{p}(X)$ consists of elements $\alpha \in C H^{p}(X)$ such that $k^{*} \alpha=k^{2 p-i} \alpha$ (which is equivalent to $k_{*} \alpha=k^{2 g-2 p+i} \alpha$ ) for all $k \in \mathbb{Z}$.

1.2. When $(X, \theta)$ is a principally polarized abelian variety, we may identify $X$ with its dual. Let $p_{i}: X \times X \rightarrow X, i=1,2$ be the two projections and $m: X \times X \rightarrow X$ the addition map. The Poincaré line bundle on $X \times X$ is represented by the divisor $P:=p_{1}^{*} \theta+p_{2}^{*} \theta-m^{*} \theta$. The Fourier transform $\mathcal{F}: C H(X) \rightarrow C H(X)$ is defined by $\mathcal{F}(\alpha)=\left(p_{2}\right)_{*}\left(p_{1}^{*} \alpha \cdot e^{P}\right)$. The morphism $\mathcal{F}$ turns out to be an automorphism of the $\mathbb{Q}$-vector space $C H(X)$. We refer to [B1] and [B2] for a detailed study of the fundamental properties of $\mathcal{F}$.

1.3. The particular case of the Jacobian $J$ of a smooth projective curve $C$ of genus $g \geq 2$ has been studied by several authors. For any point $x_{0} \in C$, we have a natural map: $\iota: C \rightarrow J$ given by $x \mapsto \mathcal{O}_{C}\left(x-x_{0}\right)$. We denote by $\mathcal{T}$ the smallest subring of $C H(J)$ containing the class $[\iota(C)]=\iota_{*}[C]$ which is closed under the pull-backs $k^{*}$ and push-forwards $k_{*}$ for all $k \in \mathbb{Z}$ and under the Fourier transform $\mathcal{F}$, which will be called the tautological subring of $C H(J)$. Notice that this ring depends on the choice of the base point $x_{0} \in C$. 
Let us consider the decomposition of $[\iota(C)]$ given by (11) (note $C H_{(-1)}^{g-1}(J)=$ $0)$ :

$$
[\iota(C)]=C_{(0)}+C_{(1)}+\ldots+C_{(g-1)} \text { where } \quad C_{(i)} \in C H_{(i)}^{g-1}(J) .
$$

Let $\theta$ be a symmetric theta divisor on $J$. As in [P2], we define $p_{i}=\mathcal{F}\left(C_{(i-1)}\right) \in C H_{(i-1)}^{i}(J)$ for $i \geq 1, q_{i}=\mathcal{F}\left(C_{(i)} \cdot \theta\right) \in C H_{(i)}^{i}(J)$ for $i \geq 0$.

Let $\simeq^{\text {alg }}$ be the algebraic equivalence. Note that $q_{i} \simeq^{a l g} 0$ for $i \geq 1$. Beauville proved in [B3] that the ring $\mathcal{T} / \simeq^{\text {alg }}$ is generated (as a subring under the intersection product) by the classes $\left(p_{i}\right)_{i \geq 1}$. Recently, Polishchuk proved in P2 (Thm 0.2 and Prop. 4.2) that $\mathcal{T}$ is generated by the classes $\left(p_{i}\right)_{i<g / 2+1}$ and $\left(q_{i}\right)_{i<(g+1) / 2}$.

1.4. The influence on $\mathcal{T} / \simeq^{a l g}$ of the gonality of the curve $C$ has been firstly studied by Colombo and van Geemen in CvG. They proved that if $C$ admits a $g_{d}^{1}$, then $p_{i}$ is algebraically equivalent to zero if $i \geq d$. Recently the second named author computed some relations between the cycles $p_{i}$ in $\mathcal{T} / \simeq^{a l g}$ for a curve admitting a base point free $g_{d}^{r}$. In [GK], van der Geer and Kouvidakis gave another proof of the main result in $\mathrm{Her}$, by using the Grothendieck-Riemann-Roch theorem. They gave simpler but equivalent relations as Don Zagier proved it. Note that Polishchuk has found universal relations in $\mathcal{T} / \simeq^{\text {alg }}(\mathrm{cf}[\mathrm{P} 1])$ and in $\mathcal{T}(\mathrm{cf}[\mathrm{P} 2])$.

We will denote by $A(r, d, g)$ the Castelnuovo number, which is:

$$
A(r, d, g)=\sum_{i=0}^{r-1} \frac{(-1)^{i}}{d-2 r+2}\left(\begin{array}{c}
i+g+r-d-2 \\
i
\end{array}\right)\left(\begin{array}{c}
d-2 r \\
r-1-i
\end{array}\right)\left(\begin{array}{c}
d-r+1-i \\
r-i
\end{array}\right) .
$$

It was explained in $\mathrm{Her}$ that if $A(r, d, g) \neq 0$ one can deduce from Colombo and van Geemen's Theorem that $p_{i} \simeq^{\text {alg }} 0$ when $i \geq d-2 r+2$. In particular, the ring $\mathcal{T} / \simeq^{\text {alg }}$ is generated by $\left(p_{i}\right)_{i \leq d-2 r+1}$.

1.5. Our aim in this note is to generalize the above results to $\mathcal{T}$. More precisely we shall prove the following:

Theorem 1. Let $C$ be a smooth projective curve which admits a base point free $g_{d}^{r}$ with $D$ being a class in $g_{d}^{r}$. Let $K$ be the canonical class of $C$. If $K$ is a multiple of $D$ in $C H^{1}(C)_{\mathbb{Q}}$ and if $A(r, d, g) \neq 0$, then the tautological ring is generated by $\left(p_{i}\right)$ and $\left(q_{i}\right)$ with $i \leq d-2 r+1$.

We present two proofs : the first follows the method in GK and uses an argument in $[\mathrm{Her}$. The second one follows the methods of [Her]. In the two proofs we use the operator defined in [P2]. In both approaches, we encounter the same difficulty which can be overcomed by assuming that $K$ is a multiple of $D$. This condition is satisfied for example for curves which are complete intersections. In this case our result is much sharper than the aforementioned result of Polishchuk. 
Some applications and refinements of this theorem are given in the last sections, where we show (see Thm. 3) that if $C$ is a curve admitting a degree $d$ map $C \rightarrow \mathbb{P}^{1}$ whose ramification points are all of order $d$, then the tautological ring is generated by $\left(p_{i}\right)_{i \leq d-1}$ and $q_{1}$. In the case of a hyperelliptic curve, we obtain a precise description of $\mathcal{T}$ (Prop. 3), which generalizes a result of Collino $([\mathrm{Co}]$, Thm. 2) on the validness of Poincaré's formula under rational equivalence.

\section{Preliminary Results}

According to Cor. 24 in $\left[\mathrm{Ma}\right.$ or to Thm. 4 in [Her], if $p_{i} \simeq^{a l g} 0$ then $p_{j} \simeq^{a l g} 0$ for $j \geq i$. We generalize this to the case of rational equivalence in this section, which will be needed in the proof of Theorem 1, Recall the following operator $\mathcal{D}$ introduced in $[\mathrm{P} 2$ :

$$
\begin{aligned}
\mathcal{D}= & \frac{1}{2} \sum_{m, n \geq 1}\left(\begin{array}{c}
m+n \\
n
\end{array}\right) p_{m+n-1} \partial_{p_{m}} \partial_{p_{n}} \\
& +\sum_{m, n \geq 1}\left(\begin{array}{c}
m+n-1 \\
n
\end{array}\right) q_{m+n-1} \partial_{q_{m}} \partial_{p_{n}}-\sum_{n \geq 1} q_{n-1} \partial_{p_{n}} .
\end{aligned}
$$

Let $\mathcal{I}_{q} \subset \mathbb{Q}[p, q]$ be the ideal generated by the elements $\left(q_{i}\right)_{i \geq 1}$, so $\mathcal{I}_{q} \simeq^{\text {alg }}$ 0 . By the expression of $\mathcal{D}$, we have $\mathcal{D}\left(\mathcal{I}_{q}\right) \subset \mathcal{I}_{q}$.

Proposition 1. Let $n$ be an integer such that $2 \leq n \leq g-1$. Suppose that $p_{n} \in \mathcal{I}_{q}$, then

i) for any $m$ such that $n \leq m \leq g, p_{m}$ is contained in the ideal generated by $\left(q_{i}\right)_{1 \leq i \leq m-1}$;

ii) the tautological ring $\mathcal{T}$ is generated by $\left(p_{i}\right)_{i \leq n-1}$ and $\left(q_{i}\right)_{i \leq n-1}$.

Proof. To prove i) we use an induction on $m$. Assume $m \geq 2$ and $p_{m} \in \mathcal{I}_{q}$, then $p_{2} p_{m} \in \mathcal{I}_{q}$. We apply the operator $\mathcal{D}$, which gives $\mathcal{D}\left(p_{2} p_{m}\right) \in \mathcal{I}_{q}$. Note that $\mathcal{D}\left(p_{2} p_{m}\right)=\left(\begin{array}{c}m+2 \\ 2\end{array}\right) p_{m+1}-q_{1} p_{m}-q_{m-1} p_{2}$, thus $p_{m+1} \in \mathcal{I}_{q}$. If we write $p_{m+1}=\sum_{i \geq 1} q_{i} A_{i}$ with $A_{i} \in \mathcal{T}$, then $A_{i}=0$ for $i \geq m+1$, since $q_{i} \in C H_{(i)}^{i}$, $p_{m+1} \in C H_{(m)}^{m+1}$ and $A_{i} \in \oplus_{j \geq 0} C H_{(j)}$.

To prove ii), we just need to prove that for any $m$ such that $n \leq m \leq g$, the class $q_{m}$ is contained in the ideal generated by $\left(q_{i}\right)_{i \leq n-1}$. Assume this is true for all $m<k$, we shall prove it for $m=k$. By i) and the hypothesis of induction, we have $p_{k}=\sum_{j=1}^{n-1} q_{j} A_{j}$ with $A_{j} \in \mathcal{T}$. Now the claim follows from the following relation in [P2]:

$$
q_{k}=\mathcal{D}\left(q_{1} p_{k}\right)+q_{1} q_{k-1}=\sum_{j=1}^{n-1} \mathcal{D}\left(q_{1} q_{j} A_{j}\right)+q_{1} q_{k-1} .
$$




\section{First Proof of the TheOREM}

We shall follow the method of [GK] coupled with arguments in [Her] to prove our main theorem.

Let $C$ be a smooth projective curve with a base-point-free linear system $g_{d}^{r}$, which gives arise to a morphism: $\gamma: C \rightarrow \mathbb{P}^{r}$. Let $Y \subset C \times \check{\mathbb{P}}^{r}$ be the subvariety $\{(p, y) \mid \gamma(p) \in y\}$. The projection $\phi: Y \rightarrow C$ is a $\mathbb{P}^{r-1}$-bundle. More precisely if we denote by $E:=\gamma^{*}\left(T^{*} \mathbb{P}^{r}\right)$, then $Y=\mathbb{P}(E)$ as a projective bundle over $C$. Recall that we have the following relative Euler sequence:

$$
0 \rightarrow \mathcal{O}_{Y} \rightarrow \phi^{*}(E) \otimes \mathcal{O}_{\phi}(1) \rightarrow T_{Y} \rightarrow \phi^{*} T_{C} \rightarrow 0 .
$$

This gives $t d\left(T_{Y}\right)=t d\left(\phi^{*} T_{C}\right) \cdot t d\left(\phi^{*}(E) \otimes \mathcal{O}_{\phi}(1)\right)=\left(1-\frac{1}{2} \phi^{*} K\right) \cdot t d\left(\phi^{*}(E) \otimes\right.$ $\left.\mathcal{O}_{\phi}(1)\right)$, where $K$ is a canonical divisor of the curve $C$.

Let us denote by $\tilde{\alpha}: Y \rightarrow \check{\mathbb{P}}^{r}$ the second projection, which is a finite morphism of degree $d$. Note that $c_{1}\left(\phi^{*}(E)\right)=\phi^{*}\left(\gamma^{*}\left(c_{1}\left(T^{*} \mathbb{P}^{r}\right)\right)\right)=-(r+$ 1) $\phi^{*} D$, where $D$ is a divisor in the linear system $g_{d}^{r}$. Furthermore the higher Chern classes of $\phi^{*}(E)$ vanish, as it is the pull-back from a vector bundle over a curve. This gives that $t d\left(\phi^{*}(E) \otimes \mathcal{O}_{\phi}(1)\right)$ is a linear function in $\phi^{*}(D)$ with coefficients in $\tilde{\alpha}^{*} C H\left(\check{\mathbb{P}}^{r}\right)$.

From now on, we will identify $\mathbb{P}^{r}$ with $\check{\mathbb{P}}^{r}$ and make use of the following self-explaining notations:

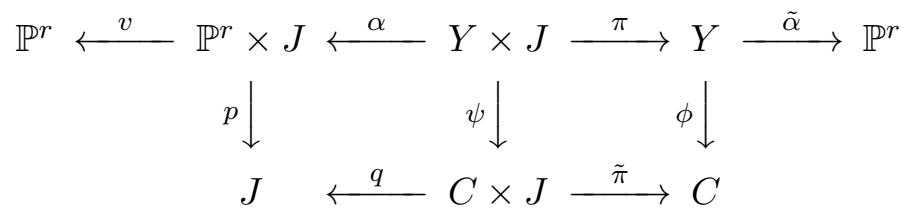

By the precedent discussions, the relative Todd class $t d(\alpha)=\pi^{*} t d(\tilde{\alpha})$ is of the form $\left(1-\frac{1}{2} \pi^{*} \phi^{*} K\right)\left(A(x)+B^{\prime}(x) \pi^{*} \phi^{*}(D)\right)=A(x)+B^{\prime}(x) \pi^{*} \phi^{*} D-$ $\frac{1}{2} A(x) \pi^{*} \phi^{*} K$, for some polynomials $A, B^{\prime}$ of degrees at most $r-1$, where $x=\alpha^{*}(\xi)$ and $\xi=v^{*}(h)$ for a hyperplane $h \subset \mathbb{P}^{r}$.

Let us denote by $\Pi$ the divisor of the Poincaré line bundle on $C \times J$ and $l:=\psi^{*}(\Pi)$. Let $L$ be the line bundle on $Y \times J$ associated to $l$. As in [GK], we apply the Grothendieck-Riemann-Roch formula to $V_{k}:=\alpha_{*}\left(L^{\otimes k}\right)$ :

$$
\operatorname{ch}\left(V_{k}\right)=\alpha_{*}\left(e^{k l} t d(\alpha)\right)=\alpha_{*}\left(e^{k l} A(x)+e^{k l} B^{\prime}(x) \pi^{*} \phi^{*} D-\frac{1}{2} e^{k l} A(x) \pi^{*} \phi^{*} K\right) .
$$

The following Lemma can be proved in a similar way as in [GK](Lemma $3.3)$.

Lemma 1. In $C H\left(\mathbb{P}^{r} \times J\right)$, we have the following relations for $\nu \geq 0$ :

$$
\alpha_{*}\left(l^{\mu} \cdot x^{\nu}\right)=\left\{\begin{array}{l}
q_{*}\left(\Pi^{\mu}\right) \xi^{\nu+1}+q_{*}\left(\Pi^{\mu} \cdot \tilde{\pi}^{*} D\right) \xi^{\nu}, \text { if } \mu>0 \\
d \xi^{\nu}, \text { if } \mu=0 .
\end{array}\right.
$$


ON THE TAUTOLOGICAL RING OF A JACOBIAN MODULO RATIONAL EQUIVALENCE

For any divisor $D_{0}$ on $C$, we have

$$
\alpha_{*}\left(l^{\mu} \cdot x^{\nu} \cdot \pi^{*} \phi^{*} D_{0}\right)=\left\{\begin{array}{l}
q_{*}\left(\Pi^{\mu} \cdot \tilde{\pi}^{*} D_{0}\right) \xi^{\nu+1}, \text { if } \mu>0 \\
\operatorname{deg}\left(D_{0}\right) \xi^{\nu+1}, \text { if } \mu=0 .
\end{array}\right.
$$

Using these formulae, one can easily obtain

$$
\begin{aligned}
\alpha_{*}\left(e^{k l} \cdot A(x)\right) & \left.=\alpha_{*}(A(x))+\sum_{\mu=1}^{\infty} \frac{k^{\mu}}{\mu !}\left(q_{*}\left(\Pi^{\mu}\right) \xi A(\xi)+q_{*}\left(\Pi^{\mu} \cdot \tilde{\pi}^{*} D\right) A(\xi)\right)\right) \\
& =d A(\xi)+q_{*}\left(e^{k \Pi}\right) \xi A(\xi)+q_{*}\left(e^{k \Pi} \cdot \tilde{\pi}^{*} D\right) A(\xi)-q_{*}\left(\tilde{\pi}^{*} D\right) A(\xi) \\
& =q_{*}\left(e^{k \Pi}\right) \xi A(\xi)+q_{*}\left(e^{k \Pi} \cdot \tilde{\pi}^{*} D\right) A(\xi) .
\end{aligned}
$$

In a similar way, one obtains $\alpha_{*}\left(e^{k l} B^{\prime}(x) \cdot \pi^{*} \phi^{*} D\right)=q_{*}\left(e^{k \Pi} \cdot \tilde{\pi}^{*} D\right) \xi B^{\prime}(\xi)$ and $\alpha_{*}\left(e^{k l} A(x) \cdot \pi^{*} \phi^{*} K\right)=q_{*}\left(e^{k \Pi} \cdot \tilde{\pi}^{*} K\right) \xi A(\xi)$.

Recall that we have fixed a base point $x_{0} \in C$ and considered the natural $\operatorname{map} \iota: C \rightarrow J$.

Lemma 2. For any $k \in \mathbb{Z}$ and any cycle $D^{\prime} \in C H(C)_{\mathbb{Q}}$, we have $\mathcal{F}\left(k_{*} \iota_{*} D^{\prime}\right)=$ $q_{*}\left(e^{k \Pi} \cdot \tilde{\pi}^{*} D^{\prime}\right)$.

Proof. We have a Cartesian diagram:

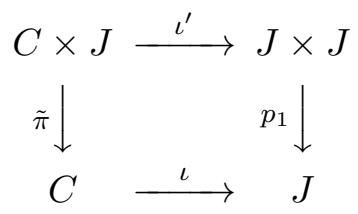

Let $q^{\prime}: J \times J \rightarrow J$ be the second projection and $P$ the Poincaré divisor on $J \times J$. We have $\mathcal{F}\left(k_{*} \iota_{*} D^{\prime}\right)=q_{*}^{\prime}\left(p_{1}^{*} k_{*} \iota_{*} D^{\prime} \cdot e^{P}\right)=q_{*}^{\prime}\left(k_{*}^{\prime} p_{1}^{*} \iota_{*} D^{\prime} \cdot e^{P}\right)=$ $q_{*}^{\prime} k_{*}^{\prime}\left(p_{1}^{*} \iota_{*} D^{\prime} \cdot e^{k P}\right)=q_{*}^{\prime}\left(\iota_{*}^{\prime} \tilde{\pi}^{*} D^{\prime} \cdot e^{k \Pi}\right)=q_{*}\left(e^{k \Pi} \cdot \tilde{\pi}^{*} D^{\prime}\right)$. Here $k^{\prime}: J \times J \rightarrow J \times J$ is given by $(x, y) \mapsto(k x, y)$.

Finally we obtain the following:

$\operatorname{ch}\left(V_{k}\right)=\mathcal{F}\left(k_{*} \iota_{*}[C]\right) \xi A(\xi)+\mathcal{F}\left(k_{*} \iota_{*} D\right)\left(A(\xi)+\xi B^{\prime}(\xi)\right)-\frac{1}{2} \mathcal{F}\left(k_{*} \iota_{*} K\right) \xi A(\xi)$.

Note that modulo algebraic equivalence, this gives exactly the formula in Prop. 3.1 in $[\mathrm{GK}]$. From now on, we assume furthermore that $K=\frac{1}{s} D$ in $C H^{1}(C)_{\mathbb{Q}}$ for some $s \in \mathbb{Q}$. Let $B(\xi)=s A(\xi)+s \xi B^{\prime}(\xi)-\frac{1}{2} \xi A(\xi)$, then we get a simpler formula for $\operatorname{ch}\left(V_{k}\right)$ as follows:

$$
\operatorname{ch}\left(V_{k}\right)=\mathcal{F}\left(k_{*} \iota_{*}[C]\right) \xi A(\xi)+\mathcal{F}\left(k_{*} \iota_{*} K\right) B(\xi) .
$$

Recall that $\mathcal{F}\left(k_{*} \iota_{*}[C]\right)=\sum_{i=1}^{g} k^{i+1} p_{i}$ and $\mathcal{F}\left(k_{*} \iota_{*} K\right)=(2 g-2)+2 \sum_{i=1}^{g} k^{i} q_{i}$. Let us write $\xi A(\xi)=\sum_{i=0}^{r-1} a_{i} \xi^{i+1}$ and $B(\xi)=\sum_{i=0}^{r} b_{i} \xi^{i}$, then a direct calculus shows:

$$
c h_{j}\left(V_{k}\right)=2 s k^{j} q_{j}+\sum_{i=1}^{j-1}\left(k^{j-i+1} a_{i-1} p_{j-i}+2 k^{j-i} b_{i} q_{j-i}\right) \xi^{i}+(2 g-2) b_{j} \xi^{j} .
$$


To simplify the notations, we will put $c h_{j}\left(V_{k}\right)=\sum_{m=0}^{j} A_{m}(j) \xi^{m}$, where $A_{m}(j)$ is of codimension $j-m$.

Note that modulo $\mathcal{I}_{q}$, the expressions of $A_{m}(j)$ are the same as those in GK]. By the proof of Prop. 3.6 and that of the main Theorem in [GK] we obtain the following generalization of the main result in [GK]:

Theorem 2. If $C$ admits a base point free $g_{d}^{r}$ with $D \in g_{d}^{r}$ and $K$ is a multiple of $D$ in $C H^{1}(C)_{\mathbb{Q}}$, then $\sum_{\alpha_{1}+\cdots+\alpha_{r}=M-2 r+1}\left(\alpha_{1}+1\right) ! \cdots\left(\alpha_{r}+\right.$ $1) ! p_{\alpha_{1}+1} \cdots p_{\alpha_{r}+1}$ is contained in $\mathcal{I}_{q}$ for all $M \geq d$.

When $r \geq 2$, we recall the Castelnuovo number:

$$
A(r, d, g)=\sum_{i=0}^{r-1} \frac{(-1)^{i}}{d-2 r+2}\left(\begin{array}{c}
i+g+r-d-2 \\
i
\end{array}\right)\left(\begin{array}{c}
d-2 r \\
r-1-i
\end{array}\right)\left(\begin{array}{c}
d-r+1-i \\
r-i
\end{array}\right) .
$$

Consider the case $M=d$ in the precedent theorem. Then the argument in section 6.2 [Her] shows that $p_{d-2 r+2}$ is contained in $\mathcal{I}_{q}$ (here we note that Cor. 0.3 in [P1], which is used in Section 6.2 [Her, holds in fact in $\mathcal{T}$ modulo $\mathcal{I}_{q}$ instead of $\mathcal{T} / \simeq^{a l g}$ ). This concludes the proof of the Theorem 1 by Prop. 1.

Remark : i) The condition in the theorem on the relationship between $K$ and $D$ can be weakened to that $\mathcal{F}\left(\iota_{*} D\right)-\operatorname{deg}(D)[J]$ is contained in $\mathcal{I}_{q}$.

ii) This method also gives a way to compute the terms in $\mathcal{I}_{q}$, which in turn gives relations in the tautological subring $\mathcal{T}$.

Examples : Let $C \subset \mathbb{P}^{r}$ be a smooth curve which is a complete intersection of hypersurfaces of degrees $\left(d_{1}, \ldots, d_{r-1}\right)$. One shows easily that $K_{C}=\left.\mathcal{O}_{\mathbb{P}^{r}}\left(\sum d_{i}-r-1\right)\right|_{C}$ which is a multiple of $D:=C \cap H \in g_{d}^{r}$ with $d=\prod d_{i}$ and $H$ is a hyperplane in $\mathbb{P}^{r}$. If $d_{1}, \ldots, d_{r-1}$ are sufficiently big, then $A(r, d, g)>0$ and Theorem 1 applies. We note that in this case $d-2 r+1$ is much smaller than $\frac{g}{2}+1$ because $g=\frac{\left(\sum d_{i}-(r+1)\right) d}{2}+1$, thus this result is sharper than that in [P2].

\section{Another proof of the Theorem}

In this section we give the sketch of a proof of Theorem 1 based upon the method described in $\mathrm{Her}$. Although this approach is different, we encounter the same difficulties as above, i.e we need a relation between $D$ and $K$ to proceed.

We will note $C_{n}$ for the $n$-th symmetric product of $C$. Suppose that $C$ carries $\mathcal{G}$ a base point free $g_{d}^{r}$ which contains $D=o_{1}+\ldots+o_{d}$. Theorem 3 in [Her] gives the class $\mathcal{G}$ in $C H\left(C_{d}\right)$ in terms of the classes of the diagonals 
ON THE TAUTOLOGICAL RING OF A JACOBIAN MODULO RATIONAL EQUIVALENCE

$$
\begin{aligned}
\delta_{i_{1}, \ldots, i_{r}}=\left\{i_{1} x_{1}+\ldots+i_{r} x_{r} \mid\left(x_{1}, \ldots, x_{r}\right) \in C^{r}\right\}: \\
{[\mathcal{G}]=\sum_{\substack{1 \leq i_{1} \leq \ldots \leq i_{r} \\
1 \leq j_{1}<\ldots<j_{s} \leq d}}\left(\prod_{u=1}^{r} \frac{(-1)^{i_{u}-1}}{i_{u}}\right)\left[\delta_{i_{1}, \ldots, i_{r}}+o_{j_{1}}+\ldots+o_{j_{s}}\right], }
\end{aligned}
$$

where $s=d-\sum_{j=1}^{r} i_{j}$.

Consider the morphism $u_{d}: C_{d} \rightarrow J$ which maps $D$ onto the class of the divisor $D-d x_{0}$. It contracts $\mathcal{G}$ onto a point in the Jacobian, so $u_{d *}[\mathcal{G}]=0$. Moreover, $u_{d *}\left[\delta_{i_{1}, \ldots, i_{r}}+o_{1}+\ldots+o_{d-\sum i_{a}}\right]$ is a multiple of $\left(i_{1} * C\right) * \ldots *\left(i_{r} * C\right) *\left[o_{1}+\ldots+o_{d-\sum i_{a}}-\left(d-\sum i_{a}\right) x_{0}\right]$. Then we use the same arguments as in the begining of section 5 in [Her], this is the decomposition (2), the relations $i_{*} C_{(a)}=i^{a+2} C_{(a)}$ and the projection onto $C H(J)_{(s)}^{g-r}$ for $s \geq 0$. We get :

$$
\begin{gathered}
\sum_{\substack{0 \leq a_{1}, \ldots, a_{r}, t \\
a_{1}+\ldots+a_{r}+t=s}} C_{\left(a_{1}\right)} * \ldots * C_{\left(a_{r}\right)} * \beta\left(d, a_{1}, \ldots, a_{r}\right)_{(t)}=0 \quad \text { where } \\
\beta\left(d, a_{1}, \ldots, a_{r}\right)=\sum_{i_{1}=1}^{d} \ldots \sum_{i_{r}=1}^{d}(-1)^{i_{1}+\ldots+i_{r}} i_{1}{ }^{a_{1}+1} \ldots i_{r}{ }^{a_{r}+1} \alpha_{d-i_{1}-\ldots-i_{r}},
\end{gathered}
$$

and where

$$
\alpha_{u}=\sum_{1 \leq k_{1}<k_{2}<\ldots<k_{u} \leq d}\left[o_{k_{1}}+\ldots+o_{k_{u}}-u x_{0}\right] .
$$

We do not know if the cycles $\alpha_{u}$ are in $\mathcal{T}$ in general. As in the previous section, we only know how to obtain relations in $\mathcal{T}$ when $D$ is a multiple of $K$, which we will suppose from now on. In this case $\alpha_{1}$ is a multiple of $\iota_{*} K$ and thus belongs to $\mathcal{T}$. Then we use an induction and the relations

$(i+1) \alpha_{i+1}=\alpha_{i} *\left(\alpha_{1}\right)-\alpha_{i-1} *\left(2_{*} \alpha_{1}\right)+\cdots+(-1)^{i-1} \alpha_{1} *\left(i_{*} \alpha_{1}\right)+(-1)^{i}(i+1)_{*} \alpha_{1}$

to prove that $\alpha_{u} \in \mathcal{T}$ for $0 \leq u \leq d$. Now let us apply $\mathcal{F}$ to (3) and deduce relations modulo $\mathcal{I}_{q}$. As the elements $\mathcal{F}\left(\alpha_{u}\right)$ are Fourier transforms of 0 cycles, they belong to $\bigoplus_{s>0}\left(\mathcal{T} \cap C H(J)_{(s)}^{s}\right)=\mathbb{Q}\left[q_{0}, q_{1}, \ldots, q_{g}\right]$ and we have $\mathcal{F}\left(\alpha_{u}\right)=\mathcal{F}\left(\alpha_{u}\right)_{(0)}\left(\bmod \mathcal{I}_{q}\right)$. Then, $\mathcal{F}\left(\beta\left(d, a_{1}, \ldots, a_{r}\right)_{(t)}\right) \in \mathcal{I}_{q}$ if $t>0$. As

$$
\mathcal{F}\left(\alpha_{u}\right)_{(0)}=\sum_{1 \leq k_{1}<\ldots<k_{u} \leq d}[J]=\left(\begin{array}{l}
d \\
u
\end{array}\right)[J],
$$

we obtain the relations

$$
\begin{gathered}
\sum_{\substack{0 \leq a_{1}, \ldots, a_{r} \\
a_{1}+\ldots+a_{r}=s}} \gamma\left(d, a_{1}, \ldots, a_{r}\right) p_{a_{1}+1} \ldots p_{a_{r}+1}=0 \quad\left(\bmod \mathcal{I}_{q}\right) \quad \text { where } \\
\gamma\left(d, a_{1}, \ldots, a_{r}\right)=\sum_{i_{1}=1}^{d} \ldots \sum_{i_{r}=1}^{d}(-1)^{i_{1}+\ldots+i_{r}}\left(\begin{array}{c}
d \\
i_{1}+\ldots+i_{r}
\end{array}\right) i^{a_{1}+1} \ldots i_{r}^{a_{r}+1} .
\end{gathered}
$$


Now according to the appendix of Don Zagier in [GK] these relations are the same as those in Theorem 2, Then using Prop 1, a similar argument as that in the precedent section concludes the proof.

\section{Curves admitting a pure Ramification}

We begin with the following easy result.

Proposition 2. Let $C$ be a smooth projective curve of genus $g$ and $x \in C$ a point such that $K_{C}=(2 g-2) x$ in $C H^{1}(C)_{\mathbb{Q}}$. Then the tautological ring $\mathcal{T}$ on $J$ is generated over $\mathbb{Q}$ by the classes $\left(p_{n}\right)_{n<g / 2+1}$ and $q_{1}$.

Proof. Let $[c]$ be the image of the canonical divisor of $C$ in $J$ and write $[c]=[o]+c_{1}+\cdots+c_{g}$ the decomposition after (10). By the proof of Prop. 4.3 in [Pol], we have $\mathcal{F}([c])=\exp \left(2 q_{1}\right)$, which gives $\mathcal{F}\left(c_{k}\right)=\left(2 q_{1}\right)^{k} / k !=$ $\mathcal{F}\left(c_{1}\right)^{k} / k$ ! . As a consequence, we obtain a Poincaré-type formula: $c_{k}=\frac{c_{1}^{* k}}{k !}$.

Now suppose that there exists a point $x \in C$ such that $K_{C}=(2 g-2) x$ in $C H^{1}(C)_{\mathbb{Q}}$. Then $\iota_{*} K=2(g-1)\left[x-x_{0}\right]$, which gives that $(2 g-2)_{*} \iota_{*} K=$ $2(g-1)[c]$. Let $\eta=\iota_{*} K / 2+[0]$ and $\eta_{n}$ the component of $\eta$ in $C H_{(n)}^{g}$. By the formula on page 4 of [P2], we have $q_{n}=\mathcal{F}\left(\eta_{n}\right)$. The precedent discussions give that $c_{k}=2(2 g-2)^{k-1} \eta_{k}$. This gives $2(2 g-2)^{k-1} q_{k}=$ $\mathcal{F}\left(c_{k}\right)=\mathcal{F}\left(c_{1}^{* k}\right) / k !=\mathcal{F}\left(c_{1}\right)^{k} / k !=\left(2^{k} / k !\right) q_{1}^{k}$, which gives

$$
q_{k}=\frac{q_{1}^{k}}{k !(g-1)^{k-1}} \text {. }
$$

By Prop. 4.2 of [Pol], we obtain the claim.

Remark : Similar argument can be applied to more general cases. For example, if $K=(g-1)(x+y)$ for some points $x, y \in C$. Then we have $\iota_{*} K=(g-1)\left(\left[x-x_{0}\right]+\left[y-x_{0}\right]\right)=\left[x+y-2 x_{0}\right] *(-1)_{*} \iota_{*} K$. Apply $(g-1)_{*}$ to both sides, we obtain $(g-1)_{*}(2 \eta-2[o])=[c] *(1-g)_{*}(2 \eta-2[o])$. This gives that

$$
[o]+\sum_{i=1}(g-1)^{i-1} \eta_{i}=\left([o]+\sum_{i=1} c_{i}\right) *\left([o]+\sum_{i=1}(-1)^{i}(g-1)^{i-1} \eta_{i}\right) .
$$

The first non-trivial relation is $2(g-1)^{2} \eta_{3}=c_{3}-\eta_{1} * c_{2}+(g-1) \eta_{2} * c_{1}$. Applying the Fourier transformation, we obtain: $2(g-1)^{2} q_{3}=-2 / 3 q_{1}^{3}+$ $2(g-1) q_{2} q_{1}$. Similarly we obtain expressions of $q_{5}, q_{7} \cdots$. This gives that the tautological ring is generated by $\left(p_{n}\right)_{n<g / 2+1}, q_{1}$ and $\left(q_{2 k}\right)_{2 k<g / 2+1}$.

We say that a curve $C$ admits a pure ramification of degree $d$ if there exists a degree $d$ map $C \rightarrow \mathbb{P}^{1}$ whose ramification points are all of order $d$. In this case, if we take $x$ to be a ramification point, then by the Hurwitz's formula, we have $K=(2 g-2) x$ in $C H^{1}(C)_{\mathbb{Q}}$. Combining with Theorem 1 . we obtain 
Theorem 3. Let $C$ be a smooth projective curve of genus $g$ which admits a pure ramification of degree $d$, then the tautological ring $\mathcal{T}$ on its Jacobian is generated by the classes $\left(p_{i}\right)_{i \leq d-1}$ and $q_{1}$.

For example, let $f_{d}(x, y)$ be a homogeneous polynomial of degree $d$ such that the plane curve $C:\left(z^{d}+f_{d}(x, y)=0\right)$ is smooth. The map $C \rightarrow \mathbb{P}^{1}$ given by $[x: y: z] \mapsto[x: y]$ gives a pure ramification of $C$, thus Theorem 3 applies.

\section{The Case of a hyperellitpic CuRve}

When $C$ is a hyperelliptic curve, we can obtain a more precise description of $\mathcal{T}$ as follows:

Proposition 3. Let $C$ be a hyperelliptic curve of genus $g$ and a the smallest integer such that $q_{1}^{a}=0$, then we have

$$
\mathcal{T}=\frac{\mathbb{Q}\left[p_{1}, q_{1}\right]}{\left(p_{1}^{g+1}, q_{1} p_{1}^{g}, \ldots, q_{1}^{a-1} p_{1}^{g-a+2}, q_{1}^{a}\right)} .
$$

Note that according to the proof of Proposition 2 the integer $a$ is the dimension of $\mathcal{T} \cap C H^{g}(J)$.

Proof. By the precedent Theorem, $\mathcal{T}$ is generated by $p_{1}$ and $q_{1}$. We present another direct proof of this as follows. We will use Proposition 1 and prove that $p_{2} \in \mathcal{I}_{q}$. Note $\mu$ for the hyperelliptic involution, and $D$ for the divisor $2 x_{0}-(p+\mu(p))$ for any $p \in C$. Then $-C$ is the translated of $C$ by $D$ in the Jacobian. In terms of cycles we get $(-1)_{*}[C]=[D] *[C]$. Let us consider $x_{1}$ a ramification point of $C$ and $\beta$ the class of the divisor $x_{1}-x_{0}$. We get $D=(-2)_{*} \beta$. But we also have $\iota_{*} K=2(g-1) \beta$. Recall that $\iota_{*} K=2(\eta-[0])$ and apply $\mathcal{F}$ to get :

$$
(-1)_{*}\left(p_{1}+p_{2}+\ldots+p_{g}\right)=\frac{1}{g-1}(-2)^{*}\left((g-1)[J]+q_{1}+q_{2}+\ldots\right) *\left(p_{1}+p_{2}+\ldots+p_{g}\right) \text {. }
$$

The relation we obtain in $C H_{(1)}^{2}(J)$ is $p_{2}=\frac{1}{g-1} p_{1} q_{1}$, so $p_{2} \in \mathcal{I}_{q}$.

We claim that for any integers $(m, n)$ such that $m<a$ and $m+n<g+1$, we have $q_{1}^{m} p_{1}^{n} \neq 0$. In fact, otherwise we can use an induction and the following formula

$$
\mathcal{D}\left(q_{1}^{m} p_{1}^{n}\right)=n(m+n-g-1) q_{1}^{m} p_{1}^{n-1}
$$

to deduce that $q_{1}^{m}=0$, which is a contradiction.

Remark : The relation $p_{2}=\frac{1}{g-1} p_{1} q_{1}$ is coherent with the relation $p_{2}=p_{1} q_{1}$ given as an exemple of Prop 4.2 in [P2] for (hyperelliptic) curves of genus 2. Note that equation (41) also gives the relations $p_{a}=\sum_{i=1}^{a-1} \frac{(-2)^{i-1}}{g-1} p_{a-i} q_{i}$ when $a$ is even, and $\sum_{i=1}^{a-1} \frac{(-2)^{i}}{g-1} p_{a-i} q_{i}=0$ when $a$ is odd.

If we choose the base point $x_{0}$ to be one of the ramification points, then 
$q_{1}=0$ and $\mathcal{T}=\frac{\mathbb{Q}\left[p_{1}\right]}{\left(p_{1}^{g+1}\right)}$, so in this case, Poincaré's formula holds modulo rational equivalence, which has been firstly proved in [Co] (Theorem 2).

\section{REFERENCES}

[B1] A. Beauville, Quelques remarques sur la transformation de Fourier dans l'anneau de Chow d'une variété abélienne, Algebraic geometry (Tokyo/Kyoto, 1982), 238-260, Lecture Notes in Math., 1016, Springer, Berlin, 1983.

[B2] A. Beauville, Sur l'anneau de Chow d'une variété abélienne, Math. Ann. 273 (1986), 647-651.

[B3] A. Beauville, Algebraic cycles on Jacobian varieties, Compos. Math. 140 (2004), no. no.3, 683-688.

[Co] A. Collino, Poincaré's formulas and hyperelliptic curves, Atti Accad. Sci. Torino 109 (1975), 89-101.

[CvG] E. Colombo and B. van Geemen, Note on curves in a Jacobian, Compositio Math. 88 (1993), no.3, 333-353.

[GK] Gerard van der Geer, Alexis Kouvidakis, Cycle relations on Jacobian varieties, math/0608606, to appear in Compos. Math.

[Her] F. Herbaut, Algebraic cycles on the Jacobian of a curve with a linear system of given dimension, arXiv:math/0606140, to appear in Compos. Math.

[Ma] G. Marini, Tautological Cycles on Jacobian Varieties, arXiv:math/0509659

[P1] A. Polishchuk, Universal algebraic equivalences between tautological cycles on Jacobians of curves, Math. Z. 251 (2005), no. 4, 875-897.

[P2] A. Polishchuk, Lie symmetries of the Chow group of a Jacobian and the tautological subring, arXiv:math/0504448, to appear in J. Alg. Geom.

C.N.R.S., Labo. J. Leray, Faculté des sciences, Université de NANTES

2, Rue de la Houssinière, BP 92208, F-44322 Nantes Cedex 03 France

fu@math.univ-nantes.fr

IUFM NICE / Laboratoire GRIM - Université de Toulon et du Var Boulevard Toussaint Merle, 83500 La Seyne Sur Mer, France

herbaut@math.unice.fr 